\title{
Lyapunov-Type Inequalities for Riemann-Liouville Type Fractional Boundary Value Problems with Fractional Boundary Conditions
}

\author{
Debananda Basua $^{a}$, Jagan Mohan Jonnalagadda ${ }^{a}$, Dipak Kumar Satpathi ${ }^{a}$ \\ a Department of Mathematics, Birla Institute of Technology and Science Pilani, Hyderabad Campus, Telangana, India.
}

\begin{abstract}
In this article, we establish Lyapunov-type inequalities for two-point Riemann-Liouville type fractional boundary value problems associated with well-posed fractional boundary conditions. To illustrate the applicability of established results, we estimate lower bounds for eigenvalues of the corresponding eigenvalue problems and deduce criteria for the nonexistence of real zeros of certain Mittag-Leffler functions.
\end{abstract}

Keywords: IRiemann-Liouville type fractional derivative boundary value problem Green's function Lyapunov-type inequality Mittag-Leffler function 2010 MSC: 34A08, 34A40, 26D10, 33E12, 34C10.

\section{Introduction}

Lyapunov [10] established a necessary condition, known as the Lyapunov inequality, for the existence of a nontrivial solution of Hill's equation associated with Dirichlet boundary conditions. This inequality has several applications in various problems related to the theory of differential equations. Due to its importance, the Lyapunov inequality has been generalized in many forms. For a detailed discussion on Lyapunov-type inequalities and their applications, we refer [2, 12, 13, 17, 19, 20] and the references therein.

Recently, many researchers have derived Lyapunov-type inequalities for various classes of fractional boundary value problems [8, 15, 16, 18, 21]. For the first time, Ferreira [5] obtained a Lyapunov-type inequality for a two-point Riemann-Liouville type fractional boundary value problem associated with Dirichlet boundary conditions as follows:

Email addresses: debananda04@gmail.com (Debananda Basua), j.jaganmohan@hotmail.com (Jagan Mohan Jonnalagadda), dipak@hyderabad.bits-pilani.ac.in (Dipak Kumar Satpathi) 
Theorem 1.1. [5] If the fractional boundary value problem

$$
\left\{\begin{array}{l}
D_{a}^{\alpha} y(t)+q(t) y(t)=0, \quad a \leq t \leq b, \\
y(a)=0, \quad y(b)=0,
\end{array}\right.
$$

has a nontrivial solution, where $q:[a, b] \rightarrow \mathbb{R}$ is a continuous nonnegative function, then

$$
\int_{a}^{b} q(s) d s>\Gamma(\alpha)\left(\frac{4}{b-a}\right)^{\alpha-1}
$$

Recently, Ntouyas et al. [11 presented a survey of results on Lyapunov-type inequalities for fractional differential equations associated with a variety of boundary conditions. This article shows a gap in the literature on Lyapunov-type inequalities for two-point Riemann-Liouville type fractional boundary value problems associated with fractional boundary conditions. In 2016, Dhar et al. 3] derived Lyapunov-type inequalities for two-point Riemann-Liouville type fractional boundary value problems associated with fractional integral boundary conditions. This article stresses the importance of choosing well-posed boundary conditions for Riemann-Liouville type fractional boundary value problems. In this line, the authors [7] have obtained Lyapunov-type inequalities for two-point Riemann-Liouville type fractional boundary value problems associated with well-posed mixed, Sturm-Liouville, Robin and general boundary conditions, recently.

Motivated by these developments, in this article, we establish Lyapunov-type inequalities for two-point Riemann-Liouville type fractional boundary value problems associated with well-posed fractional boundary conditions.

\section{Preliminaries}

Throughout, we shall use the following notations, definitions and known results of fractional calculus [9, 14]. Denote the set of all real numbers and complex numbers by $\mathbb{R}$ and $\mathbb{C}$, respectively.

Definition 2.1. [9] The Euler gamma function is defined by

$$
\Gamma(z):=\int_{0}^{\infty} t^{z-1} e^{-t} d t, \quad \Re(z)>0 .
$$

Using the reduction formula

$$
\Gamma(z+1)=z \Gamma(z), \quad \Re(z)>0,
$$

the Euler gamma function can be extended to the half-plane $\Re(z) \leq 0$ except for $z \neq 0,-1,-2, \ldots$

Definition 2.2. [9] Let $\alpha>0$ and $a \in \mathbb{R}$. The $\alpha^{\text {th }}$-order Riemann-Liouville fractional integral of a function $y:[a, b] \rightarrow \mathbb{R}$ is defined by

$$
I_{a}^{\alpha} y(t)=\frac{1}{\Gamma(\alpha)} \int_{a}^{t}(t-s)^{\alpha-1} y(s) d s, \quad a \leq t \leq b,
$$

provided the right-hand side exists. For $\alpha=0$, define $I_{a}^{\alpha}$ to be the identity map. Moreover, let $n$ denote a positive integer and assume $n-1<\alpha \leq n$. The $\alpha^{\text {th }}$-order Riemann-Liouville fractional derivative is defined as

$$
D_{a}^{\alpha} y(t)=D^{n} I_{a}^{n-\alpha} y(t), \quad a \leq t \leq b,
$$

where $D^{n}$ denotes the classical $n^{\text {th }}$-order derivative, if the right-hand side exists.

Definition 2.3. [9] We denote by $L(a, b)$ the space of Lebesgue measurable functions $y:[a, b] \rightarrow \mathbb{R}$ for which

$$
\|y\|_{L}=\int_{a}^{b}|y(t)| d t<\infty .
$$


Definition 2.4. [9] We denote by $C[a, b]$ the space of continuous functions $y:[a, b] \rightarrow \mathbb{R}$ with the norm

$$
\|y\|_{C}=\max _{t \in[a, b]}|y(t)| .
$$

Lemma 2.5. [9] If $\alpha \geq 0$ and $\beta>0$, then

$$
\begin{aligned}
I_{a}^{\alpha}(t-a)^{\beta-1} & =\frac{\Gamma(\beta)}{\Gamma(\beta+\alpha)}(t-a)^{\beta+\alpha-1}, \\
D_{a}^{\alpha}(t-a)^{\beta-1} & =\frac{\Gamma(\beta)}{\Gamma(\beta-\alpha)}(t-a)^{\beta-\alpha-1} .
\end{aligned}
$$

Lemma 2.6. [9] Let $\alpha>\beta>0$ and $y \in C[a, b]$. Then,

$$
D_{a}^{\beta} I_{a}^{\alpha} y(t)=I_{a}^{\alpha-\beta} y(t), \quad t \in[a, b] .
$$

Lemma 2.7. [1] Let $\alpha>0$ and $n$ be a positive integer such that $n-1<\alpha \leq n$. Then, the fractional differential equation

$$
D_{a}^{\alpha} y(t)=0, \quad a<t<b,
$$

has a unique solution $y \in C(a, b) \cap L(a, b)$, and is given by

$$
y(t)=C_{1}(t-a)^{\alpha-1}+C_{2}(t-a)^{\alpha-2}+\cdots+C_{n}(t-a)^{\alpha-n},
$$

where $C_{i} \in \mathbb{R}, i=1,2, \cdots, n$.

Lemma 2.8. [1] Let $\alpha>0$ and $n$ be a positive integer such that $n-1<\alpha \leq n$. If $y \in C(a, b) \cap L(a, b)$, then

$$
I_{a}^{\alpha} D_{a}^{\alpha} y(t)=y(t)+C_{1}(t-a)^{\alpha-1}+C_{2}(t-a)^{\alpha-2}+\cdots+C_{n}(t-a)^{\alpha-n}
$$

for some $C_{i} \in \mathbb{R}, i=1,2, \cdots, n$.

\section{Main Results}

In this section, we obtain two Lyapunov-type inequalities for the fractional boundary value problem

$$
\left\{\begin{array}{l}
D_{a}^{\alpha} y(t)+q(t) y(t)=0, \quad a \leq t \leq b \\
y(a)=0, D_{a}^{\beta} y(b)=0
\end{array}\right.
$$

using the properties of the corresponding Green's function.

Theorem 3.1. Let $1<\alpha \leq 2,0 \leq \beta \leq 1$ such that $0<(\alpha-\beta)<1$ and $h:[a, b] \rightarrow \mathbb{R}$. Then, the fractional boundary value problem

$$
\left\{\begin{array}{l}
D_{a}^{\alpha} y(t)+h(t)=0, \quad a \leq t \leq b \\
y(a)=0, D_{a}^{\beta} y(b)=0
\end{array}\right.
$$

has the unique solution

$$
y(t)=\int_{a}^{b} G(t, s) h(s) d s
$$

where

$$
G(t, s)=\left\{\begin{array}{lc}
\frac{1}{\Gamma(\alpha)}\left(\frac{b-s}{b-a}\right)^{\alpha-\beta-1}(t-a)^{\alpha-1}, & a \leq t \leq s<b \\
\frac{1}{\Gamma(\alpha)}\left[\left(\frac{b-s}{b-a}\right)^{\alpha-\beta-1}(t-a)^{\alpha-1}-(t-s)^{\alpha-1}\right], & a \leq s \leq t<b
\end{array}\right.
$$


Proof. Applying $I_{a}^{\alpha}$ on both sides of (3) and using Lemma 2.8, we have

$$
y(t)=-I_{a}^{\alpha} h(t)+C_{1}(t-a)^{\alpha-1}+C_{2}(t-a)^{\alpha-2},
$$

for some $C_{1}, C_{2} \in \mathbb{R}$. Applying $D_{a}^{\beta}$ on both sides of (5), using Lemma 2.5 and Lemma 2.6, we get

$$
\begin{aligned}
D_{a}^{\beta} y(t) & =-I_{a}^{\alpha-\beta} h(t)+C_{1} \frac{\Gamma(\alpha)}{\Gamma(\alpha-\beta)}(t-a)^{\alpha-\beta-1} \\
& +C_{2} \frac{\Gamma(\alpha-1)}{\Gamma(\alpha-\beta-1)}(t-a)^{\alpha-\beta-2} .
\end{aligned}
$$

Using $y(a)=0$ in (5), we get $C_{2}=0$. Using $D_{a}^{\beta} y(b)=0$ in (6), we get

$$
C_{1}=\frac{1}{(b-a)^{\alpha-\beta-1} \Gamma(\alpha)} \int_{a}^{b}(b-s)^{\alpha-\beta-1} h(s) d s .
$$

Substituting $C_{1}$ and $C_{2}$ in (5), the unique solution of (3) is

$$
\begin{aligned}
y(t) & =-\frac{1}{\Gamma(\alpha)} \int_{a}^{t}(t-s)^{\alpha-1} h(s) d s \\
& +\frac{(t-a)^{\alpha-1}}{(b-a)^{\alpha-\beta-1} \Gamma(\alpha)} \int_{a}^{b}(b-s)^{\alpha-\beta-1} h(s) d s \\
& =\frac{1}{\Gamma(\alpha)} \int_{a}^{t}\left[\left(\frac{b-s}{b-a}\right)^{\alpha-\beta-1}(t-a)^{\alpha-1}-(t-s)^{\alpha-1}\right] h(s) d s \\
& +\frac{1}{\Gamma(\alpha)} \int_{t}^{b}\left(\frac{b-s}{b-a}\right)^{\alpha-\beta-1}(t-a)^{\alpha-1} h(s) d s \\
& =\int_{a}^{b} G(t, s) h(s) d s .
\end{aligned}
$$

The proof is complete.

Corollary 3.2. Let $1<\alpha \leq 2,0 \leq \beta \leq 1$ such that $1 \leq(\alpha-\beta)<2$. Then, the fractional boundary value problem (3) has the unique solution

$$
y(t)=\int_{a}^{b} G(t, s) h(s) d s
$$

where

$$
G(t, s)=\left\{\begin{array}{lc}
\frac{1}{\Gamma(\alpha)}\left(\frac{b-s}{b-a}\right)^{\alpha-\beta-1}(t-a)^{\alpha-1}, & a \leq t \leq s \leq b, \\
\frac{1}{\Gamma(\alpha)}\left[\left(\frac{b-s}{b-a}\right)^{\alpha-\beta-1}(t-a)^{\alpha-1}-(t-s)^{\alpha-1}\right], & a \leq s \leq t \leq b .
\end{array}\right.
$$

Proof. The proof is similar to the proof of Theorem 3.1 .

Remark 3.3. Recently, Eloe et al. [4] have obtained the Green's function for

$$
-D_{0}^{\alpha} y=0, \quad a \leq t \leq b,
$$

satisfying the boundary conditions

$$
y(0)=D_{0}^{\beta} y(b)=0 .
$$

Now, we prove that these Green's functions are nonnegative and obtain upper bounds for both the Green's functions and their integrals.

Theorem 3.4. The Green's function $G(t, s)$ for Theorem 3.1 satisfies

$$
G(t, s) \geq 0, \text { for }(t, s) \in[a, b) \times[a, b) .
$$


Proof. For $a \leq t \leq s<b$,

$$
G(t, s)=\frac{1}{\Gamma(\alpha)}\left(\frac{b-s}{b-a}\right)^{\alpha-\beta-1}(t-a)^{\alpha-1} \geq 0 .
$$

Now, suppose $a \leq s \leq t<b$. Then, we have

$$
\left(\frac{b-s}{b-a}\right)^{\alpha-\beta-1} \geq 1 \text { and }(t-s)^{\alpha-1} \leq(t-a)^{\alpha-1},
$$

implying that

$$
\begin{aligned}
G(t, s) & =\frac{1}{\Gamma(\alpha)}\left[\left(\frac{b-s}{b-a}\right)^{\alpha-\beta-1}(t-a)^{\alpha-1}-(t-s)^{\alpha-1}\right] \\
& \geq \frac{1}{\Gamma(\alpha)}\left[(t-a)^{\alpha-1}-(t-s)^{\alpha-1}\right] \geq 0 .
\end{aligned}
$$

The proof is complete.

Corollary 3.5. The Green's function $G(t, s)$ for Corollary 3.2 satisfies

$$
G(t, s) \geq 0, \text { for }(t, s) \in[a, b] \times[a, b] .
$$

Proof. For $a \leq t \leq s \leq b$,

$$
G(t, s)=\frac{1}{\Gamma(\alpha)}\left(\frac{b-s}{b-a}\right)^{\alpha-\beta-1}(t-a)^{\alpha-1} \geq 0 .
$$

Now, suppose $a \leq s \leq t \leq b$. Since

$$
(t-a)(b-s)-(b-a)(t-s)=(s-a)(b-t) \geq 0 \text { and }(t-a) \geq(t-s),
$$

we have

$$
(t-a)^{\alpha-\beta-1}(b-s)^{\alpha-\beta-1} \geq(b-a)^{\alpha-\beta-1}(t-s)^{\alpha-\beta-1}
$$

and

$$
(t-a)^{\beta} \geq(t-s)^{\beta},
$$

implying that

$$
\begin{aligned}
G(t, s) & =\frac{1}{\Gamma(\alpha)}\left[\left(\frac{b-s}{b-a}\right)^{\alpha-\beta-1}(t-a)^{\alpha-1}-(t-s)^{\alpha-1}\right] \\
& =\frac{\left[(b-s)^{\alpha-\beta-1}(t-a)^{\alpha-1}-(b-a)^{\alpha-\beta-1}(t-s)^{\alpha-1}\right]}{(b-a)^{\alpha-\beta-1} \Gamma(\alpha)} \\
& =\frac{(t-a)^{\beta}\left[(t-a)^{\alpha-\beta-1}(b-s)^{\alpha-\beta-1}\right]}{(b-a)^{\alpha-\beta-1} \Gamma(\alpha)} \\
& -\frac{(t-s)^{\beta}\left[(b-a)^{\alpha-\beta-1}(t-s)^{\alpha-\beta-1}\right]}{(b-a)^{\alpha-\beta-1} \Gamma(\alpha)} \\
& \geq \frac{(t-a)^{\beta}\left[(t-a)^{\alpha-\beta-1}(b-s)^{\alpha-\beta-1}\right]}{(b-a)^{\alpha-\beta-1} \Gamma(\alpha)} \\
& -\frac{(t-a)^{\beta}\left[(b-a)^{\alpha-\beta-1}(t-s)^{\alpha-\beta-1}\right]}{(b-a)^{\alpha-\beta-1} \Gamma(\alpha)} \geq 0 .
\end{aligned}
$$

The proof is complete. 
Theorem 3.6. For the Green's function $G(t, s)$ defined in (4),

$$
\max _{t \in[a, b]} \frac{G(t, s)}{(b-s)^{\alpha-\beta-1}}=\frac{G(s, s)}{(b-s)^{\alpha-\beta-1}}, \quad s \in[a, b],
$$

and

$$
\max _{s \in[a, b]} \frac{G(s, s)}{(b-s)^{\alpha-\beta-1}}=\frac{(b-a)^{\beta}}{\Gamma(\alpha)} .
$$

Proof. First, we show that for any fixed $s \in[a, b], \frac{G(t, s)}{(b-s)^{\alpha-\beta-1}}$ increases from $\frac{G(a, s)}{(b-s)^{\alpha-\beta-1}}$ to $\frac{G(s, s)}{(b-s)^{\alpha-\beta-1}}$, and then decreases to $\frac{G(b, s)}{(b-s)^{\alpha-\beta-1}}$. Let $a \leq t \leq s<b$ and consider

$$
\frac{\partial}{\partial t}\left[\frac{G(t, s)}{(b-s)^{\alpha-\beta-1}}\right]=\frac{1}{(b-a)^{\alpha-\beta-1} \Gamma(\alpha-1)}(t-a)^{\alpha-2}>0,
$$

implying that $\frac{G(t, s)}{(b-s)^{\alpha-\beta-1}}$ is an increasing function of $t$. Now, suppose $a \leq s \leq t<b$ and consider

$$
\begin{aligned}
\frac{\partial}{\partial t}\left[\frac{G(t, s)}{(b-s)^{\alpha-\beta-1}}\right] & =\frac{1}{\Gamma(\alpha-1)}\left[\frac{(t-a)^{\alpha-2}}{(b-a)^{\alpha-\beta-1}}-\frac{(t-s)^{\alpha-2}}{(b-s)^{\alpha-\beta-1}}\right] \\
& \leq \frac{(t-a)^{\alpha-2}}{\Gamma(\alpha-1)}\left[\frac{1}{(b-a)^{\alpha-\beta-1}}-\frac{1}{(b-s)^{\alpha-\beta-1}}\right] \leq 0,
\end{aligned}
$$

implying that $\frac{G(t, s)}{(b-s)^{\alpha-\beta-1}}$ is a decreasing function of $t$. Thus, we have

$$
\max _{t \in[a, b]} \frac{G(t, s)}{(b-s)^{\alpha-\beta-1}}=\frac{G(s, s)}{(b-s)^{\alpha-\beta-1}}, \quad s \in[a, b] .
$$

Clearly,

$$
\max _{s \in[a, b]} \frac{G(s, s)}{(b-s)^{\alpha-\beta-1}}=\max _{s \in[a, b]} \frac{(s-a)^{\alpha-1}}{(b-a)^{\alpha-\beta-1} \Gamma(\alpha)}=\frac{(b-a)^{\beta}}{\Gamma(\alpha)} .
$$

The proof is complete.

Corollary 3.7. For the Green's function $G(t, s)$ defined in (7),

$$
\max _{t \in[a, b]} G(t, s)=G(s, s), \quad s \in[a, b]
$$

and

$$
\max _{s \in[a, b]} G(s, s)=\frac{(b-a)^{\alpha-1}}{2^{2 \alpha-\beta-2} \Gamma(\alpha)} .
$$

Proof. First, we show that for any fixed $s \in[a, b], G(t, s)$ increases from $G(a, s)$ to $G(s, s)$, and then decreases to $G(b, s)$. Let $a \leq t \leq s \leq b$ and consider

$$
\frac{\partial}{\partial t} G(t, s)=\frac{1}{\Gamma(\alpha-1)}\left(\frac{b-s}{b-a}\right)^{\alpha-\beta-1}(t-a)^{\alpha-2}>0
$$

implying that $G(t, s)$ is an increasing function of $t$. Now, suppose $a \leq s \leq t \leq b$. We have

$$
(b-s)^{\alpha-\beta-1} \leq(b-a)^{\alpha-\beta-1} \text { and }(t-s)^{\alpha-2} \geq(t-a)^{\alpha-2},
$$

implying that

$$
\begin{aligned}
\frac{\partial}{\partial t} G(t, s) & =\frac{1}{\Gamma(\alpha-1)}\left[\left(\frac{b-s}{b-a}\right)^{\alpha-\beta-1}(t-a)^{\alpha-2}-(t-s)^{\alpha-2}\right] \\
& =\frac{(t-a)^{\alpha-2}(b-s)^{\alpha-\beta-1}-(t-s)^{\alpha-2}(b-a)^{\alpha-\beta-1}}{(b-a)^{\alpha-\beta-1} \Gamma(\alpha-1)} \leq 0,
\end{aligned}
$$


implying that $G(t, s)$ is a decreasing function of $t$. Thus, we have

$$
\max _{t \in[a, b]} G(t, s)=G(s, s), \quad s \in[a, b] .
$$

To prove the second part, consider

$$
G(s, s)=\frac{(s-a)^{\alpha-1}(b-s)^{\alpha-\beta-1}}{(b-a)^{\alpha-\beta-1} \Gamma(\alpha)} .
$$

Differentiating $G(s, s)$ with respect to $s$ and equating it to 0 , we obtain $s=\frac{a+b}{2}$. Again, differentiating $G^{\prime}(s, s)$ with respect to $s$, we observe that $G^{\prime \prime}(s, s) \leq 0$ at $s=\frac{a+b}{2}$. So, $G(s, s)$ attains its maximum at $s=\frac{a+b}{2}$. The proof is complete.

Corollary 3.8. For the Green's functions $G(t, s)$ defined in (4) and (7),

$$
\max _{t \in[a, b]} \int_{a}^{b} G(t, s) d s=\frac{(\alpha-1)^{\alpha-1}}{\Gamma(\alpha+1)}\left(\frac{b-a}{\alpha-\beta}\right)^{\alpha} .
$$

Proof. Consider

$$
\begin{aligned}
\int_{a}^{b} G(t, s) d s & =\frac{1}{\Gamma(\alpha)} \int_{a}^{t}\left[\left(\frac{b-s}{b-a}\right)^{\alpha-\beta-1}(t-a)^{\alpha-1}-(t-s)^{\alpha-1}\right] d s \\
& +\frac{1}{\Gamma(\alpha)} \int_{t}^{b}\left(\frac{b-s}{b-a}\right)^{\alpha-\beta-1}(t-a)^{\alpha-1} d s \\
& =\frac{(b-a)(t-a)^{\alpha-1}}{(\alpha-\beta) \Gamma(\alpha)}-\frac{(t-a)^{\alpha}}{\Gamma(\alpha+1)}
\end{aligned}
$$

Take

$$
f(t)=\frac{(b-a)(t-a)^{\alpha-1}}{(\alpha-\beta) \Gamma(\alpha)}-\frac{(t-a)^{\alpha}}{\Gamma(\alpha+1)}, \quad t \in[a, b] .
$$

Differentiating $f(t)$ with respect to $t$ and equating it to 0 , we obtain

$$
t=a+\frac{(\alpha-1)(b-a)}{(\alpha-\beta)}=A
$$

Again, differentiating $f^{\prime}(t)$ with respect to $t$, we observe that $f^{\prime \prime}(t) \leq 0$ at $t=A$. So, $f(t)$ attains its maximum at $t=A$. The proof is complete.

Remark 3.9. Recently, Hollon et al. [6] have also obtained Corollary 3.8.

We are now able to formulate Lyapunov-type inequalities for the fractional boundary value problem (3).

Theorem 3.10. Let $1<\alpha \leq 2,0 \leq \beta \leq 1$ such that $0<(\alpha-\beta)<1$. If the following fractional boundary value problem

$$
\left\{\begin{array}{l}
D_{a}^{\alpha} y(t)+q(t) y(t)=0, \quad a \leq t \leq b, \\
y(a)=0, D_{a}^{\beta} y(b)=0
\end{array}\right.
$$

has a nontrivial solution, then

$$
\int_{a}^{b}(b-s)^{\alpha-\beta-1}|q(s)| d s>\frac{\Gamma(\alpha)}{(b-a)^{\beta}} .
$$


Proof. Let $\mathfrak{B}=C[a, b]$ be the Banach space of functions endowed with norm

$$
\|y\|_{C}=\max _{t \in[a, b]}|y(t)| .
$$

It follows from Theorem 3.1 that a solution to 8 satisfies the equation

$$
y(t)=\int_{a}^{b} G(t, s) q(s) y(s) d s .
$$

Hence,

$$
\begin{aligned}
\|y\|_{C} & =\max _{t \in[a, b]}\left|\int_{a}^{b} G(t, s) q(s) y(s) d s\right| \\
& \leq \max _{t \in[a, b]}\left[\int_{a}^{b} G(t, s)|q(s)||y(s)| d s\right] \\
& \leq\|y\|_{C}\left[\max _{t \in[a, b]} \int_{a}^{b} G(t, s)|q(s)| d s\right] \\
& \leq\|y\|_{C}\left[\max _{(t, s) \in[a, b] \times[a, b]} \frac{G(t, s)}{(b-s)^{\alpha-\beta-1}}\right] \int_{a}^{b}(b-s)^{\alpha-\beta-1}|q(s)| d s,
\end{aligned}
$$

or, equivalently,

$$
1<\left[\max _{(t, s) \in[a, b] \times[a, b]} \frac{G(t, s)}{(b-s)^{\alpha-\beta-1}}\right] \int_{a}^{b}(b-s)^{\alpha-\beta-1}|q(s)| d s .
$$

An application of Theorem 3.6 yields the result.

Corollary 3.11. Let $1<\alpha \leq 2,0 \leq \beta \leq 1$ such that $1 \leq(\alpha-\beta)<2$. If the fractional boundary value problem (8) has a nontrivial solution, then

$$
\int_{a}^{b}|q(s)| d s>\frac{\Gamma(\alpha) 2^{2 \alpha-\beta-2}}{(b-a)^{\alpha-1}} .
$$

Proof. Let $\mathfrak{B}=C[a, b]$ be the Banach space of functions endowed with norm

$$
\|y\|_{C}=\max _{t \in[a, b]}|y(t)| .
$$

It follows from Corollary 3.2 that a solution to (8) satisfies the equation

$$
y(t)=\int_{a}^{b} G(t, s) q(s) y(s) d s .
$$

Hence,

$$
\begin{aligned}
\|y\|_{C} & =\max _{t \in[a, b]}\left|\int_{a}^{b} G(t, s) q(s) y(s) d s\right| \\
& \leq \max _{t \in[a, b]}\left[\int_{a}^{b} G(t, s)|q(s)||y(s)| d s\right] \\
& \leq\|y\|_{C}\left[\max _{t \in[a, b]} \int_{a}^{b} G(t, s)|q(s)| d s\right] \\
& \leq\|y\|_{C}\left[\max _{(t, s) \in[a, b] \times[a, b]} G(t, s)\right] \int_{a}^{b}|q(s)| d s,
\end{aligned}
$$

or, equivalently,

$$
1<\left[\max _{(t, s) \in[a, b] \times[a, b]} G(t, s)\right] \int_{a}^{b}|q(s)| d s .
$$

An application of Corollary 3.7 yields the result. 


\section{Applications}

In this section, we discuss two applications of Theorem 3.10 and Corollary 3.11. First, we estimate lower bounds for the eigenvalues of the Riemann-Liouville type fractional eigenvalue problems corresponding to (8).

Theorem 4.1. Let $1<\alpha \leq 2,0 \leq \beta \leq 1$ such that $0<(\alpha-\beta)<1$. Assume that $y$ is a nontrivial solution of the Riemann-Liouville type fractional eigenvalue problem

$$
\left\{\begin{array}{l}
D_{a}^{\alpha} y(t)+\lambda y(t)=0, \quad a \leq t \leq b \\
y(a)=0, D_{a}^{\beta} y(b)=0
\end{array}\right.
$$

where $y(t) \neq 0$ for each $t \in(a, b)$. Then,

$$
|\lambda|>\frac{(\alpha-\beta) \Gamma(\alpha)}{(b-a)^{\alpha}}
$$

Corollary 4.2. Let $1<\alpha \leq 2,0 \leq \beta \leq 1$ such that $1 \leq(\alpha-\beta)<2$. Assume that $y$ is a nontrivial solution of the Riemann-Liouville type fractional eigenvalue problem (11) where $y(t) \neq 0$ for each $t \in(a, b)$. Then,

$$
|\lambda|>\frac{\Gamma(\alpha) 2^{2 \alpha-\beta-2}}{(b-a)^{\alpha}} .
$$

Consider the two-parameter Mittag-Leffler function [9]

$$
E_{\alpha, \beta}(z)=\sum_{k=0}^{\infty} \frac{z^{k}}{\Gamma(k \alpha+\beta)}, \quad z, \beta \in \mathbb{C}, \quad \Re(\alpha)>0 .
$$

As the second application, we use Theorem 3.10 and Corollary 3.11 to obtain an interval in which the Mittag-Leffler function (14) has no real zeros.

Theorem 4.3. Let $1<\alpha \leq 2,0 \leq \beta \leq 1$ such that $0<(\alpha-\beta)<1$. Then, the Mittag-Leffler function $E_{\alpha, \alpha-\beta}(x)$ has no real zeros for

$$
|x| \leq(\alpha-\beta) \Gamma(\alpha) .
$$

Proof. Let $a=0, b=1$ and consider the fractional boundary value problem

$$
\left\{\begin{array}{l}
D_{0}^{\alpha} y(t)+\lambda y(t)=0, \quad 0 \leq t \leq 1 \\
y(0)=0, D_{0}^{\beta} y(1)=0 .
\end{array}\right.
$$

By Corollary 5.1 of [9], the general solution of the fractional differential equation

$$
D_{0}^{\alpha} y(t)+\lambda y(t)=0
$$

is given by

$$
y(t)=c_{1} t^{\alpha-1} E_{\alpha, \alpha}\left(-\lambda t^{\alpha}\right)+c_{2} t^{\alpha-2} E_{\alpha, \alpha-1}\left(-\lambda t^{\alpha}\right), \quad t \in(0,1] .
$$

Denote by

$$
g(t)=t^{\alpha-1} E_{\alpha, \alpha}\left(-\lambda t^{\alpha}\right)=t^{\alpha-1} \sum_{n=0}^{\infty} \frac{(-\lambda)^{n} t^{\alpha n}}{\Gamma(\alpha n+\alpha)} .
$$

Then

$$
g^{\prime}(t)=t^{\alpha-2} \sum_{n=0}^{\infty} \frac{(-\lambda)^{n} t^{\alpha n}}{\Gamma(\alpha n+\alpha-1)}=t^{\alpha-2} E_{\alpha, \alpha-1}\left(-\lambda t^{\alpha}\right) .
$$


Note that

$$
\begin{aligned}
D_{0}^{\beta} g(t) & =\sum_{n=0}^{\infty} \frac{(-\lambda)^{n}}{\Gamma(\alpha n+\alpha)} D_{0}^{\beta} t^{\alpha n+\alpha-1} \\
& =\sum_{n=0}^{\infty} \frac{(-\lambda)^{n}}{\Gamma(\alpha n+\alpha)} \frac{\Gamma(\alpha n+\alpha)}{\Gamma(\alpha n+\alpha-\beta)} t^{\alpha n+\alpha-\beta-1} \\
& =\sum_{n=0}^{\infty} \frac{(-\lambda)^{n}}{\Gamma(\alpha n+\alpha-\beta)} t^{\alpha n+\alpha-\beta-1}=t^{\alpha-\beta-1} E_{\alpha, \alpha-\beta}\left(-\lambda t^{\alpha}\right) .
\end{aligned}
$$

Also, note that

$$
g(0)=0
$$

Using $y(0)=0$ in (16), we get $c_{2}=0$. Using $D_{0}^{\beta} y(1)=0$ in 16$)$, we get that the eigenvalues $\lambda \in \mathbb{R}$ of 15 are the solutions of

$$
E_{\alpha, \alpha-\beta}(-\lambda)=0,
$$

and the corresponding eigenfunctions are given by

$$
y(t)=t^{\alpha-1} E_{\alpha, \alpha}\left(-\lambda t^{\alpha}\right), \quad t \in[0,1] .
$$

By Theorem 3.10, if a real eigenvalue $\lambda$ of 15 exists, i.e. $\lambda$ is a zero of (17), then

$$
|\lambda|>(\alpha-\beta) \Gamma(\alpha) .
$$

The proof is complete.

Corollary 4.4. Let $1<\alpha \leq 2,0 \leq \beta \leq 1$ such that $1 \leq(\alpha-\beta)<2$. Then, the Mittag-Leffler function $E_{\alpha, \alpha-\beta}(x)$ has no real zeros for

$$
|x| \leq 2^{2 \alpha-\beta-2} \Gamma(\alpha) .
$$

Proof. The proof is similar to the proof of Theorem 4.3 .

\section{References}

[1] Z. Bai and H. Lü, Positive solutions for boundary value problem of nonlinear fractional differential equation, J. Math. Anal. Appl., 311 (2005), 495 - 505.

[2] R.C. Brown and D.B. Hinton, Lyapunov Inequalities and Their Applications, In: Survey on Classical Inequalities (Ed. T.M. Rassias), Math. Appl. 517, Kluwer Acad. Publ., Dordrecht - London, 1 - 25, 2000.

[3] S. Dhar, Q. Kong and M. McCabe, Fractional boundary value problems and Lyapunov-type inequalities with fractional integral boundary conditions, Electronic Journal of Qualitative Theory of Differential Equations, 2016 (2016), No. 43, 1 16.

[4] P.W. Eloe, J.W. Lyons and J.T. Neugebauer, An ordering on Green's functions for a family of two-point boundary value problems for fractional differential equations, Communications in Applied Analysis 19 (2015), 453 - 462.

[5] R.A.C. Ferreira, A Lyapunov-type inequality for a fractional boundary value problem, Fract. Calc. Appl. Anal., 16 (2013), No. $4,978-984$.

[6] C.A. Hollon and J.T. Neugebauer, Positive solutions of a fractional boundary value problem with a fractional derivative boundary condition, Discrete Contin. Dyn. Syst. 2015, Dynamical systems, differential equations and applications. 10th AIMS Conference. Suppl., 615-620.

[7] Jagan Mohan Jonnalagadda and Debananda Basua, Lyapunov-type inequalities for two-point Riemann-Liouville type fractional boundary value problems, Novi Sad journal o Mathematics, to appear.

[8] S. Kamal, Z. Salman and A.K. Rahmat, Multiplicity Results of Multi-Point Boundary Value Problem of Nonlinear Fractional Differential Equations, Appl. Math. Inf. Sci. 12( 4)(2018), 1 - 8.

[9] A.A. Kilbas, H.M. Srivastava and J.J. Trujillo, Theory and Applications of Fractional Differential Equations, Elsevier, Amsterdam, 2006.

[10] A. Liapounoff, Problème général de la stabilité du mouvement, Ann. Fac. Sci. Toulouse Sci. Math. Sci. Phys., (2) (1907), No. 9, $203-474$. 
[11] S.K. Ntouyas, B. Ahmad and T.P. Horikis, Recent developments of Lyapunov-type inequalities for fractional differential equations, arXiv:1804.10760v1 [math.CA] 28 Apr 2018.

[12] B.G. Pachpatte, On Lyapunov type inequalities for certain higher order differential equations, J. Math. Anal. Appl., 195 (1995), No. 2, 527 - 536.

[13] J.P. Pinasco, Lyapunov-type Inequalities with Applications to Eigenvalue Problems, Springer Briefs in Mathematics, Springer, New York, 2013.

[14] I. Podlubny, Fractional Differential Equations, Academic Press, San Diego, 1999.

[15] J. Rong and C.Z. Bai, Lyapunov-type inequality for a fractional differential equation with fractional boundary conditions, Adv. Difference Equ., 2015 (2015), 10 Pages.

[16] A. Sajjad, S. Kamal, J. Fahd, On stable iterative solutions for a class of boundary value problem of nonlinear fractional order differential equations, Mathematical Methods in the Applied Sciences, (2018).

[17] A. Tiryaki, Recent developments of Lyapunov-type inequalities, Adv. Dyn. Syst. Appl., 5 (2010), No. 2,231 - 248.

[18] Y. Wang, S. Liang and C. Xia, A Lyapunov-type inequality for a fractional differential equation under Sturm-Liouville boundary conditions, Math. Inequal. Appl., 20 (2017), No. 1, 139 - 148.

[19] X. Yang, Y. Kim and K. Lo, Lyapunov-type inequality for a class of even-order linear differential equations, Appl. Math. Comput., 245 (2014), 145 - 151.

[20] X. Yang, Y. Kim and K. Lo, Lyapunov-type inequalities for a class of higher-order linear differential equations, Appl. Math. Lett., 34 (2014), 86 - 89.

[21] A. Zeeshan, Z. Akbar, S. Kamal, On UlamâĂŹs Stability for a Coupled Systems of Nonlinear Implicit Fractional Differential Equations, Bull. Malays. Math. Sci. Soc. https:doi.org/10.1007/s40840-018-0625-X. 